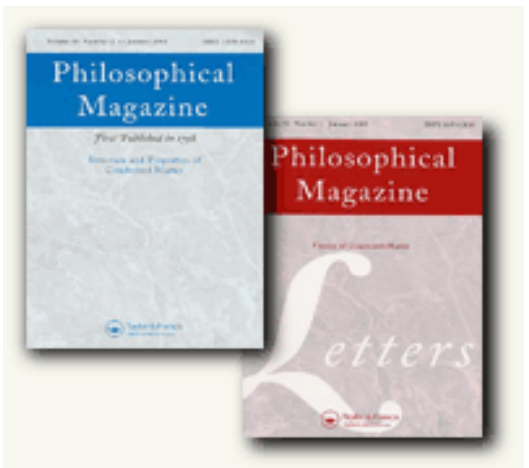

\title{
Rescaled potentials for transition metal solutes in a-iron
}

\begin{tabular}{|c|c|}
\hline Journal: & Philosophical Magazine \& Philosophical Magazine Letters \\
\hline Manuscript ID: & TPHM-09-Jun-0244.R1 \\
\hline Journal Selection: & Philosophical Magazine \\
\hline $\begin{array}{r}\text { Date Submitted by the } \\
\text { Author: }\end{array}$ & 24-Aug-2009 \\
\hline Complete List of Authors: & $\begin{array}{l}\text { Hepburn, Derek; University of Edinburgh, School of Physics and } \\
\text { Astronomy } \\
\text { Ackland, Graeme; The University of Edinburgh, School of Physics } \\
\text { and Astronomy; The University of Edinburgh, Department of } \\
\text { Physics } \\
\text { Olsson, Pär; EDF - R\&D, Département M.M.C. }\end{array}$ \\
\hline Keywords: & $\begin{array}{l}\text { modelling, molecular dynamics, transition metals, Fe-based alloys, } \\
\text { ab initio }\end{array}$ \\
\hline Keywords (user supplied): & Finnis-Sinclair, multi-component \\
\hline
\end{tabular}

\section{S) ScholaroNE \\ Manuscript Central}




\title{
Rescaled potentials for transition metal solutes in $\alpha$-iron
}

Philosophical Magazine

Vol. 00, No. 00, 00 Month 200x, 1-18

\section{RESEARCH ARTICLE}

\author{
D.J. Hepburn ${ }^{\mathrm{a} *}$, G.J. Ackland ${ }^{\mathrm{a}}$ and P. Olsson ${ }^{\mathrm{b}}$
}

aSchool of Physics and Astronomy, University of Edinburgh, James Clerk Maxwell Building, King's Buildings, Mayfield Road, Edinburgh EH9 3JZ, United Kingdom;

${ }^{\text {b} D e ́ p a r t e m e n t ~ M M C, ~ E D F-R \& D, ~ L e s ~ R e n a r d i e ̀ r e s, ~ F-77250 ~ M o r e t ~ s u r ~ L o i n g, ~ F r a n c e ~}$

\begin{abstract}
We present semi-empirical potentials for dilute transition metal solutes in $\alpha$-iron. They are in the Finnis-Sinclair form and are therefore suitable for billion atom molecular dynamics simulations. The potentials have been developed using a rescaling technique to provide soluteiron and solute-solute interactions from an existing iron potential. By fitting to first principles calculations, which show clear trends in the properties of transition metal solutes in iron across the series, we find trends in the rescaling parameters which we model using simple functions of the occupancy of the d-electron band. We comment on the possibility of utilising such relationships to the fundamental electronic properties of the solute to create multi-component potentials for transition metal solutes in $\alpha$-iron.
\end{abstract}

Keywords: Finnis-Sinclair ; semi-empirical potential ; transition metal solute ; iron ; rescaling ; multi-component

\section{Introduction}

The ability to model many component alloys of iron on the atomic level would provide an extremely powerful tool for research into the behaviour of these materials. In particular it would allow the effects of varying proportions of solutes on the properties of these materials to be studied in detail. The theoretical insights gained would complement the already extensive understanding of such systems found from experiment and ultimately impact on the design of new materials to suit particular applications such as those for the nuclear industry.

In principle one would wish to model these materials using ab-initio electronic structure calculations but the computational cost of such techniques is prohibitive, being restricted to a few hundred atoms and pico-second timescales. Alternative

${ }^{*}$ Corresponding author. Email: dhepburn@ph.ed.ac.uk 
higher level modelling techniques exist, such as kinetic monte-carlo (kMC) and molecular dynamics simulations (MD) using semi-empirical potentials, that remove these restrictions but at the expense of requiring input from experiment or abinitio calculations to fix their free parameters. Of particular relevance here, semiempirical potentials allow billion atom simulations to be performed over nanosecond timescales. The results from such simulations are readily used as an integral part of a multiscale modelling strategy and ultimately in the virtual design of new materials.

The Finnis-Sinclair (FS) scheme [1] is based around the idea of a second moment model to the local density of states. In this model the band energy depends on the width of the band, the shape of the band, and the occupation of the band. The moments theorem [2] shows how band width can be determined from the sum of squares of hopping integrals, which forms the physical basis for the cohesive term in Finnis-Sinclair potentials. The band shape and occupation are implicitly assumed to be constant.

For elements in a single phase with charge neutrality [3], the $d$-band occupation is essentially constant, and the band shape does not change massively. This underlies the success of single-element potentials. Fitting to alloys has a more troubled history. Whereas isoelectronic alloys for isostructural elements work well [4], potentials for systems involving structural phase transitions or elements from different series tend to have poor transferability from the composition at which they are fitted. Potentials covering wide ranges of concentration are, however, possible [5] as are multi-component systems [6] although the inclusion of physics beyond the second moment approximation may be necessary in general. Difficulties also arise in the Finnis-Sinclair and related schemes such as the embedded atom method (EAM) [7-9] due to the increasing complexity of the model. For an $N$-component system the number of parametrised functions grows as $N^{2}$, as does the number of data points required to fit the parameters of these functions.

There is one important exception where the band shape and electron density are reasonably constant, and we might hope that the second moment approach will work. That is multi-component alloys with one dominant element and multiple minority elements, a particularly relevant case here being ferritic steel. The dominant element fixes both the crystal structure and the electron density and should, in principle, connect the behaviour of single solute atoms to that of the dominant element and introduce stronger connections between solute-solute interactions and the properties of single solutes.

In this paper we present results of first principles calculations of the interactions between solute atoms in iron and under-coordinated and over-coordinated defects (vacancies and interstitials). We hope that these will be representative of environments encountered in molecular dynamics. We show that these calculations give clear trends with atomic number. Using these results as fitting data, we derive semi-empirical potentials for single transition metal solutes in $\alpha$-iron by rescaling the functions of a pure iron potential. We also investigate ways to connect the interactions between solute particles to those of single solute atoms in iron which would allow multi-component potentials to be built once the interactions of single solutes in iron are known. We then discuss the results for single solute atoms in iron and present the findings of our investigation into solute-solute interactions. Finally we present our conclusions. 


\section{Rescaling}

\subsection{Ab-initio calculations: Is rescaling credible?}

If the key physics of substitutional atoms in steel is such that a rescaling approach will work, we should expect that the rescaling will involve the $d$-electron density and the principal quantum number. Such an approach should work both for the perfect lattice and for defects. The properties of substitutional transition elements in Fe, in particular their magnetic character, have long been known to have systematic trends $[10,11]$. Here we supplement this work with an emphasis on total energy calculations for substitutional atoms and their interactions with point defects in bcc Fe.

We perform spin-polarised electronic structure calculations using the VASP code [12] with projector augmented wave (PAW) pseudopotentials [13] and the generalised gradient approximation [14] with the Vosko-Wilk-Nusair interpolation [15], which we find to give the best compromise between computation speed and accuracy. This gives a lattice parameter for pure iron of $2.83 \AA$, which was used in the impurity and defect calculations to define a fixed-volume supercell. Supercells of $128 \pm 1$ atoms were used with a Monkhorst-Pack $3 \times 3 \times 3$ k-point grid sampling the Brillouin zone. The energy cutoff was set to $300 \mathrm{eV}$. Full details of the calculations will be published elsewhere [16].

Ab-initio total energy calculations with configurations containing a single solute atom (see FIG. 1) show that there are systematic trends across the transition metal series for the free-atom substitutional energy $\left(E_{\mathrm{s}}\right)$, excess pressure at fixed equilibrium volume from a single solute atom $(P=-\partial U / \partial V)$, first nearest neighbour solute-iron separation $\left(r_{1 \mathrm{nn}}\right)$, binding energies of a single solute to a vacancy defect at first and second neighbour sites, $\left(E_{\mathrm{b}}^{\mathrm{V}, 1 \mathrm{nn}}\right.$ and $\left.E_{\mathrm{b}}^{\mathrm{V}, 2 \mathrm{nn}}\right)$, separations and the binding energies to a $\langle 110\rangle$-self-interstitial defect in the mixed $\left(E_{\mathrm{b}}^{\mathrm{SI}, \mathrm{M}}\right)$, compressive $\left(E_{\mathrm{b}}^{\mathrm{SI}, \mathrm{C}}\right)$, and tensile sites $\left(E_{\mathrm{b}}^{\mathrm{SI}, \mathrm{T}}\right)$, as shown in FIG. 2. We define the free-atom substitutional energy as the formation energy of a configuration containing a single solute atom from pure bcc iron and a free solute atom. To avoid the difficulties associated with the ab-initio treatment of the free atom we calculate this value as the sum of the ab-initio result for the formation energy from the pure equilibrium phase of the solute and the experimental value for the (negative) cohesive energy of the pure phase [17]. We use the following definition for the binding energy of $n$ defects and impurities, $\left\{A_{i}\right\}$ :

$$
E_{\mathrm{b}}\left(\left\{A_{i}\right\}\right)=\left[\sum_{i=1}^{n} E\left(A_{i}\right)\right]-\left[E\left(\left\{A_{i}\right\}\right)+(n-1) E_{0}\right],
$$

where $E\left(A_{i}\right)$ is the energy for a configuration containing $A_{i}$ only, $E\left(\left\{A_{i}\right\}\right)$ refers to a configuration containing all the interacting entities and $E_{0}$ refers to a configuration containing no defects or impurities i.e. bulk $\alpha$-iron.

We plot all values against the number of $d$-electrons in the free atom. In the solid this number will be affected by $s-d$ transfer of approximately 0.5 electrons per atom. Thus although there are clearly different trends with the $3 \mathrm{~d}$ solutes for morethan or less-than half filled bands, rigorously defining which material corresponds 


\subsection{Rescaling Strategy}

The starting point for our rescaling strategy is the pure iron FS-type potential of Ackland et al. [18]. We have chosen this iron potential over those from more recent works [19-21] because it reproduces many of the properties of iron from a small set of parameters and a simple choice for its component functions e.g. using the standard square root form for the embedding function. We believe that such a choice provides a more stringent means of testing our rescaling strategy and in demonstrating its efficacy.

The most general form for the energy, $U$, of an FS-type potential is given by

$$
\begin{aligned}
U\left(\left\{r_{a b}\right\}\right) & =\sum_{a, b>a} V^{\left(X_{a}, X_{b}\right)}\left(r_{a b}\right)-\sum_{a} \sqrt{\rho_{a}}, \\
\rho_{a} & =\sum_{b \neq a} \phi^{\left(X_{a}, X_{b}\right)}\left(r_{a b}\right)
\end{aligned}
$$

where $V^{\left(X_{a}, X_{b}\right)}, \phi^{\left(X_{a}, X_{b}\right)}$ and $F^{\left(X_{a}\right)}$ are parametrised functions dependent on the atomic species, $X_{a}$ and $X_{b}$. The cross-species pair functions are taken to be symmetrical here, i.e. $V^{(X, Y)} \equiv V^{(Y, X)}$ when $X \neq Y$, as are the functions, $\phi^{(X, Y)}$. 
4

5

8

9

10

11

12

13

14

15

16

17

18

19

20

21

22

23

24

25

26

27

28

29

30

31

32

33

34

35

36

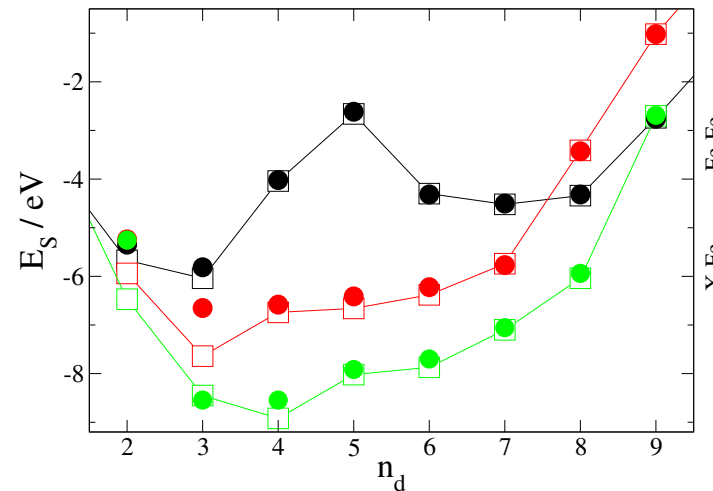

(a)

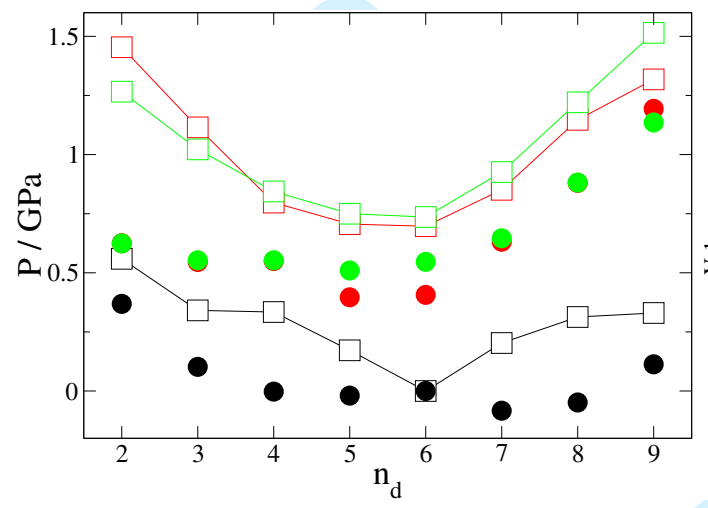

(c)

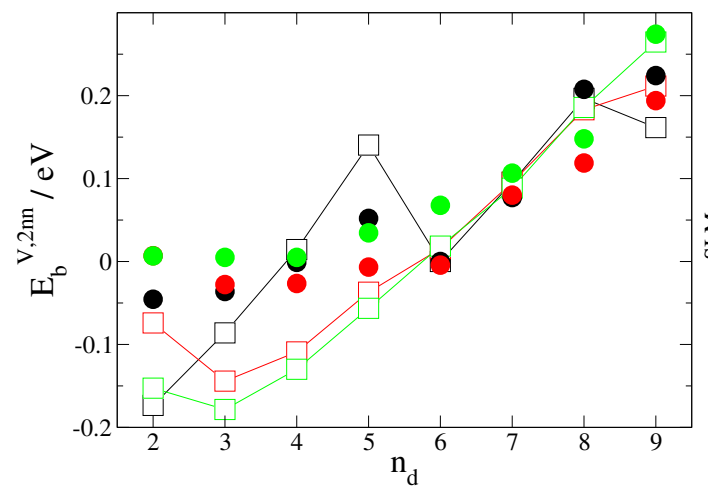

(e)

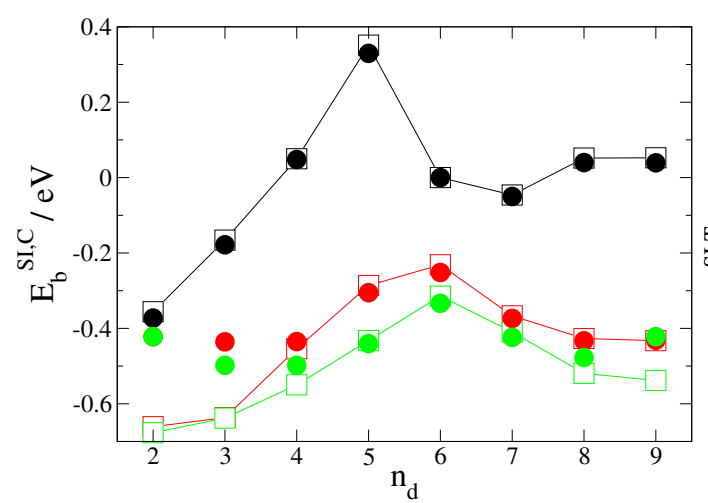

(g)

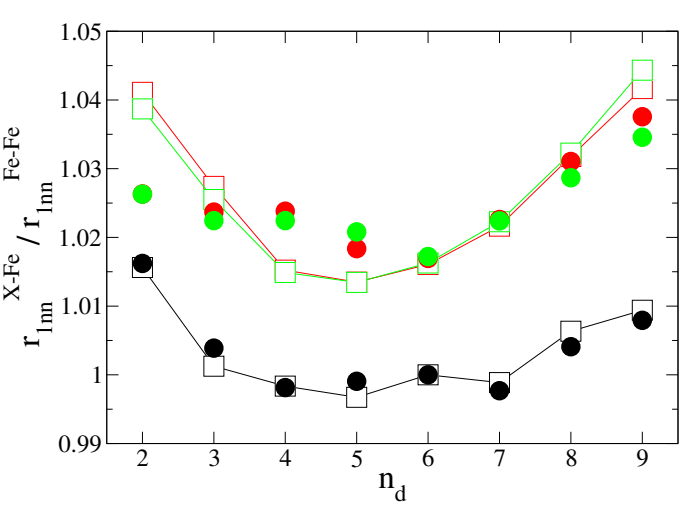

(b)

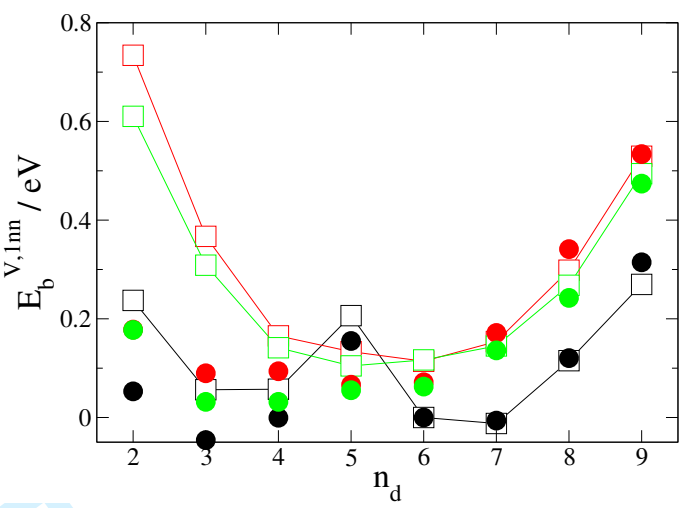

(d)

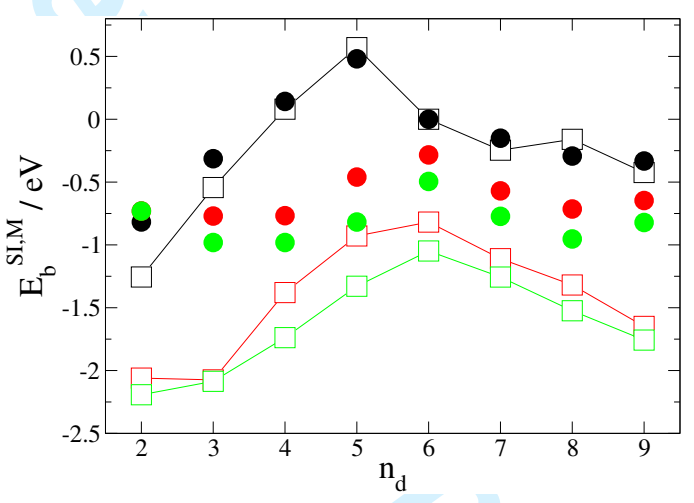

(f)

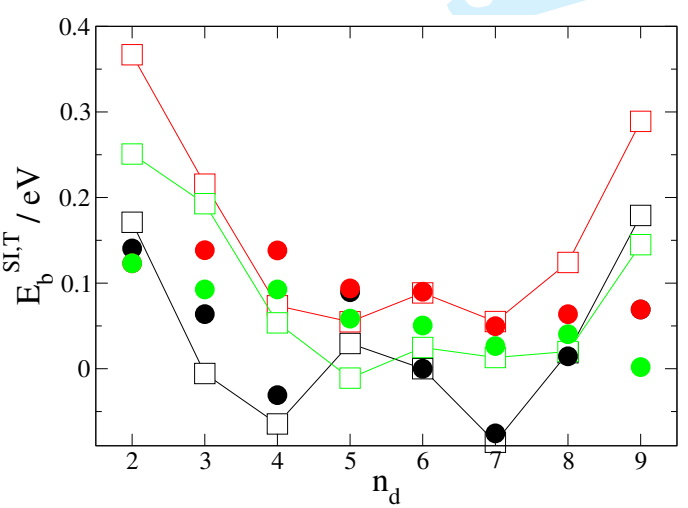

(h)

Figure 2. Fit targets (squares and lines), derived from our ab-initio data, and corresponding values from

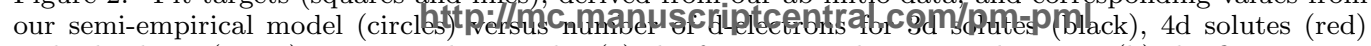
and 5d solutes (green). In particular we plot (a) the free atom substitutional energy, (b) the first nearest neighbour solute-iron separation, $r_{1 \mathrm{nn}}^{X-\mathrm{Fe}}$ relative to the corresponding iron-iron separation, $r_{1 \mathrm{nn}}^{\mathrm{Fe}-\mathrm{Fe}},(\mathrm{c})$ the excess solute pressure, the solute-vacancy binding energy at (d) 1 nn separation and (e) 2 nn separation and 
6

We use the same forms for the component functions of our potential as used in the pure iron potential [18]. In particular we define the pair functions by

$$
V^{(X, Y)}(r)= \begin{cases}\frac{Z_{X} Z_{Y} e^{2}}{4 \pi \epsilon_{0} r} \xi\left(r / r_{s}\right) & r \leq r_{1} \\ \exp \left(B_{0}+B_{1} r+B_{2} r^{2}+B_{3} r^{3}\right) & r_{1}<r \leq r_{2} \\ C^{(X, Y)}(r)=\sum_{k=1}^{6} a_{k}^{(X, Y)}\left(r_{k}^{(X, Y)}-r\right)^{3} H\left(r_{k}^{(X, Y)}-r\right) & r>r_{2}\end{cases}
$$

where $Z_{X}$ is the atomic number of species $X, r_{s}=0.88534 a_{b} / \sqrt{Z_{X}^{2 / 3}+Z_{Y}^{2 / 3}}, a_{b}$ is the Bohr radius and

$$
\xi(x)=0.1818 e^{-3.2 x}+0.5099 e^{-0.9423 x}+0.2802 e^{-0.4029 x}+0.02817 e^{-0.2016 x} .
$$

The functional form used below $r_{1}=0.9 \AA$ is the universal screened potential of Biersack and Ziegler [22], above $r_{2}=1.9 \AA^{\circ}$ it is a parametrised cubic spline with spline points, $\left\{r_{k}^{(X, Y)}\right\}$, spline coefficients, $\left\{a_{k}^{(X, Y)}\right\}$, and cutoffs implemented by the use of Heaviside step functions, $H$, and between these is an interpolating function that ensures continuity of the function and its derivative. The short range terms were not present in the original Finnis-Sinclair approach, and have a significant effect only when atoms get extremely close together, for example the primary knock-on atom of a radiation damage event. The Biersack-Zeigler function is defined for all elements, and is not subject to rescaling.

The $\phi$ functions take the form of a simple cubic spline

$$
\phi^{(X, Y)}(r)=\sum_{k=1}^{2} A_{k}^{(X, Y)}\left(R_{k}^{(X, Y)}-r\right)^{3} H\left(R_{k}^{(X, Y)}-r\right) .
$$

For the pure iron component functions i.e. $V^{(\mathrm{Fe}, \mathrm{Fe})}$ and $\phi^{(\mathrm{Fe}, \mathrm{Fe})}$ we take the parameters directly from [18]. Iron-solute interactions are defined by rescaling these two functions using rescale parameters, $\left\{p_{i}^{(X)}\right\}$ :

$$
\begin{aligned}
C^{(\mathrm{Fe}, X)}\left(p_{1}^{(X)} r\right) & =p_{2}^{(X)} C^{(\mathrm{Fe}, \mathrm{Fe})}(r) \\
\phi^{(\mathrm{Fe}, X)}\left(p_{3}^{(X)} r\right) & =p_{4}^{(X)} \phi^{(\mathrm{Fe}, \mathrm{Fe})}(r)
\end{aligned}
$$

This is equivalent to a direct rescaling of the length and energy units of the cubic spline functions given by, for example,

$$
\begin{aligned}
& r_{k}^{(\mathrm{Fe}, X)}=p_{1}^{(X)} r_{k}^{(\mathrm{Fe}, \mathrm{Fe})} \\
& a_{k}^{(\mathrm{Fe}, X)}=\frac{p_{2}^{(X)}}{p_{1}^{(X)^{3}}} a_{k}^{(\mathrm{Fe}, \mathrm{Fe})} .
\end{aligned}
$$

We take the rescaling factors, $\left\{p_{i}^{(X)}\right\}$, to be the adjustable parameters for the purposes of fitting. The trends in the fit target data should therefore translate to trends in these rescale parameters across the transition metal series. In fact it 


\section{Page 7 of 19 \\ Philosophical Magazine \& Philosophical Magazine Letters}

should be possible to quantify these trends by finding functional forms that relate the rescale parameters to the elementary electronic properties of the solutes. We present our results for these rescale parameters in the following section.

Solute-solute interactions can be defined by a similar rescaling procedure:

$$
\begin{aligned}
C^{(X, Y)}\left(p_{1}^{(X, Y)} r\right) & =p_{2}^{(X, Y)} C^{(\mathrm{Fe}, \mathrm{Fe})}(r) \\
\phi^{(X, Y)}\left(p_{3}^{(X, Y)} r\right) & =p_{4}^{(X, Y)} \phi^{(\mathrm{Fe}, \mathrm{Fe})}(r) .
\end{aligned}
$$

In principle it should be possible to relate these rescale parameters back to those for the solute-iron interactions and ultimately back to the electronic properties of the solutes. In the Finnis-Sinclair approach the $\phi$ functions are related to the tight binding hopping integrals and the band filling. Across a row in the periodic table the "hopping integral" is an average over bonds involving s- and d- wavefunctions, which have a similar functional form for all elements, scaled by atomic number. The band filling, obviously, varies monotonically across the row. Thus we can expect the functions for adjacent elements to be similar. For the $V$ functions, the long range part is mainly due to the screened coulombic potential: in the case of dilute alloys considered here, this screening arises from the electronic structure imposed by iron. The energy also depends on the ionic charge. The short range repulsion is determined by the extent of the atomic-like core electrons: again a full core shell has a size which scales with atomic number.

Thus all the ingredients of the potential depend ultimately on the atomic number, and some form of scaling with this (or, equivalently, the number of d-electrons) seems plausible. If such a relationship were found it would allow multi-component alloys to be immediately modelled. We present results for the rescale parameters, $\left\{p_{i}^{(X, X)}\right\}$, and investigate the presence of any relationships between them and the iron-solute rescale parameters, $\left\{p_{i}^{(X)}\right\}$, in what follows.

\section{Single solute interactions in iron}

In order to determine the rescale parameters, $\left\{p_{i}^{(X)}\right\}$, we fit to the ab-initio data shown in FIG. 2 for transition metal solutes in $\alpha$-iron. Initial attempts found that the mixed dumbbell $E_{\mathrm{b}}^{\mathrm{SI}, \mathrm{M}}$ could not be reproduced accurately along with the other fit targets for the $4 \mathrm{~d}$ and $5 \mathrm{~d}$ solutes. We chose to remove it as a fit target and found that it still retained its generally repulsive character with a binding energy lower than that of the compressive site. This is a satisfactory compromise as long as it is sufficiently unstable to remain unsampled in any simulation of the solid. We also chose to remove it as a fit target for the $3 \mathrm{~d}$ solutes: its accurate reproduction there is an indication of good transferability.

The fitting procedure was accomplished by minimising a least squares response function, $\chi^{2}$, of the fit parameters, $\left\{p_{i}\right\}$, given in terms of the fit targets, $\left\{t_{r}\right\}$, 
model values, $\left\{m_{r}\left(\left\{p_{i}\right\}\right)\right\}$, and weight factors, $\left\{\sigma_{r}\right\}$, by

$$
\chi^{2}\left(\left\{p_{i}\right\}\right)=\sum_{r}\left(\frac{m_{r}\left(\left\{p_{i}\right\}\right)-t_{r}}{\sigma_{r}}\right)^{2}
$$

Our potential model values were all calculated in atomically relaxed $4 \times 4 \times 4$ bcc unit cell configurations, i.e. 128 atoms before the introduction of defects and solutes, at the equilibrium volume for the pure iron potential, i.e. $a_{0}=2.8665 \AA$ [18]. This was done in order to appropriately match the ab-initio fit target data. We chose weight factors of $0.01 \mathrm{eV}$ for energies, $0.005 \mathrm{~A}^{\circ}$ for lengths and $5 \times 10^{-4} \mathrm{eV} / \mathrm{A}^{\circ}$ for pressures in our fits (or $1 \%$ of the fit target value if that was larger). The minimisation was accomplished using a local direct pattern search minimisation algorithm [23] which is particularly useful when derivative information is time consuming to calculate, untrustworthy or non-existent. We have found this is a more trustworthy method when including particle separations for relaxed configurations in the fitting procedure. Initial parameter values for the direct search algorithm were found by evaluating the response function on a regularly spaced grid of points in parameter space and choosing the lowest one. In this way we try to ensure that our minimisation strategy finds the lowest minimum of the response function; there are usually many local minima.

The fitted rescale parameters are given in FIG. 3 and TABLE. B1. It is immediately clear that there are trends across the series, especially for the $4 \mathrm{~d}$ and $5 \mathrm{~d}$ transition metal solutes. We have captured these trends by fitting simple piecewise polynomial functions to the rescale parameters, the results of which are given in EQ. B1 and shown in FIG. 3. It is difficult to say if the form these trends take can be attributed to some aspect of the underlying physics (e.g. band filling) or just a result of the rescaling procedure.

What is clear, however, is that the rescale parameters for manganese $\left(3 \mathrm{~d}, n_{d}=5\right)$ show anomalous behaviour, consistent with the anomalous properties of the solute fitting targets. Also clear is that the value of $p_{4}^{(\mathrm{Hf})}\left(5 \mathrm{~d}, n_{d}=2\right)$ is inconsistent with the trends shown. The presence of multiple minima in the response function could account for this finding but this does not seem the case for hafnium. Attempts to modify $p_{4}^{(\mathrm{Hf})}$ to be consistent with the trend resulted in a very poor response function value and a further refit returned the rescale parameter to its previous value.

Looking at FIG. 2 we can see that the trends in the rescale parameters translate to the potential model values themselves. There is especially good reproduction of the substitution energy across all three series. The binding energy, $E_{\mathrm{b}}^{\mathrm{SI}, \mathrm{C}}$, is reproduced almost as well but shows slight deviation from the ab-initio target data at the ends of the series and especially for low $n_{d}$. This is all the more impressive because the reproduction of this fit target was complicated by the onset of instability in the configuration, resulting from the absence of a stable energy minimum for some subset of parameter space. For small solutes, e.g. Mn, this resulted in the formation of a $\langle 111\rangle$ mixed interstitial upon relaxation of the configuration. For large solutes, e.g. $\mathrm{W}$, the configuration reverted to a $\langle 111\rangle$ self-interstitial interacting with the solute at approximately $\left(0,0, a_{0}\right)$ separation. Despite these complications, potentials were found that reproduced the target values well and stabilised the configuration. 


\section{Page 9 of 19}

2

4

5

9

10

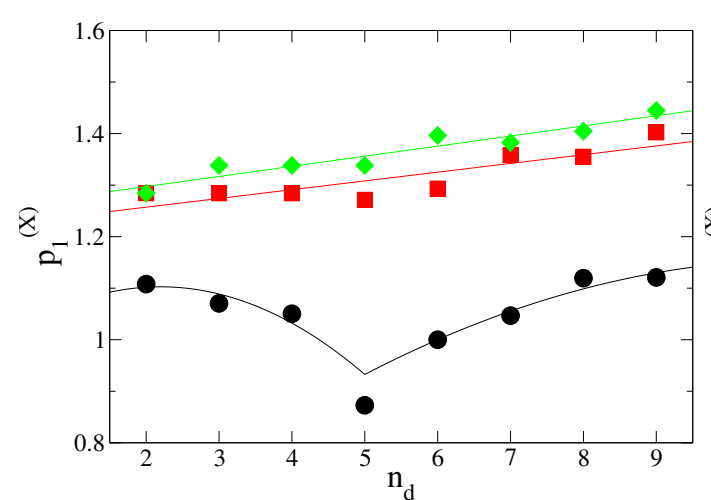

(a)

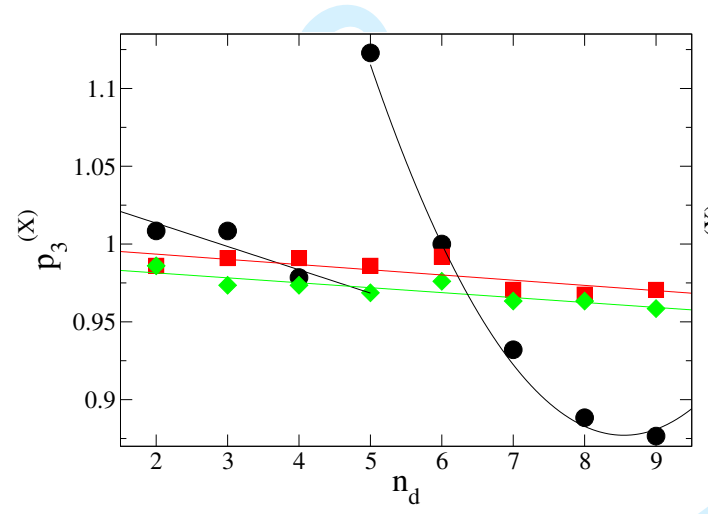

(c)

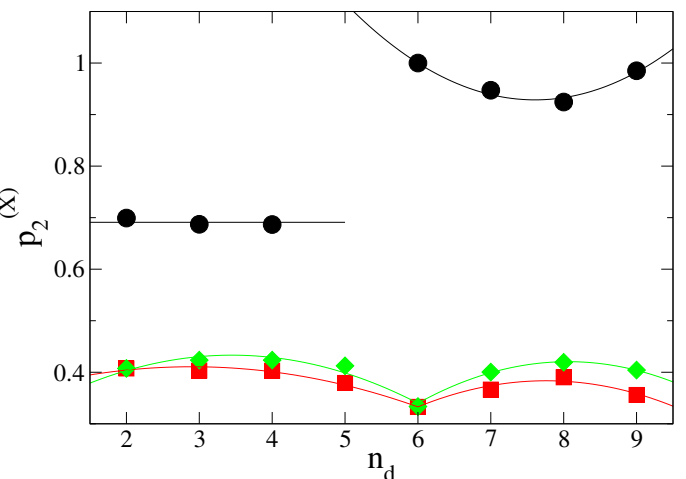

(b)

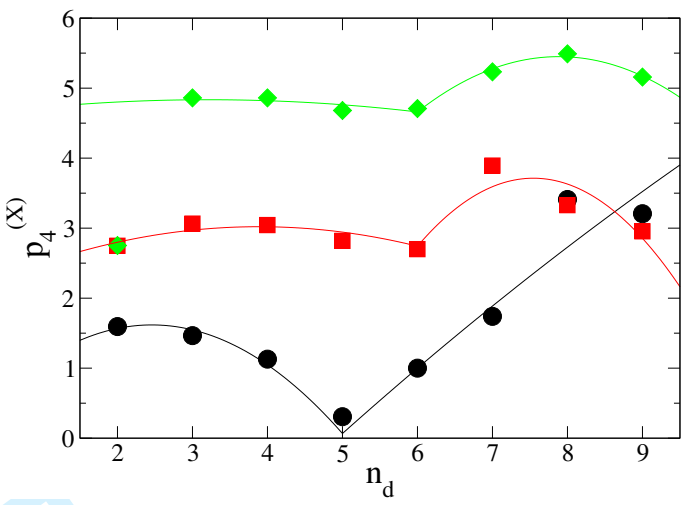

(d)

Figure 3. Rescale parameters as a function of the number of d-electrons for the 3d solutes (black circles and lines), $4 \mathrm{~d}$ solutes (red squares and lines) and $5 \mathrm{~d}$ solutes (green diamonds and lines). The graphs are for (a) $p_{1}^{(X)}$, (b) $p_{2}^{(X)}$, (c) $p_{3}^{(X)}$ and (d) $p_{4}^{(X)}$. The lines have been produced by fitting the rescale parameters to simple piecewise polynomial functions (see EQ. B1). Colour versions are available online.

Deviations from the parabolic trends in the ab-initio data are seen generally for the other fit targets. The most notable is for the binding energy, $E_{\mathrm{b}}^{\mathrm{SI}, \mathrm{T}}$, where the potential models show approximately linear behaviour in $n_{d}$. The overall deviations and the quality of the fits is best quantified via the response function values, as shown in TABLE. B3. It is clear from this data that the potentials for the low $n_{d}$ solutes perform especially poorly. For these elements d-bonding is weakest: sbonding is neglected in the Finnis-Sinclair concept but may play an important role here. It is also clear from the response function data that the $3 \mathrm{~d}$ solute potentials perform better overall than those of the other two series despite the presence of complex magnetic interactions. Even the anomalous properties of manganese are reproduced well. The excess solute pressures are, however, significantly underestimated although this is true of the $4 \mathrm{~d}$ and $5 \mathrm{~d}$ elements also.

As our only unfitted data point, it is worth returning to the difficulties experienced in fitting the binding energy of the mixed interstitial, $E_{\mathrm{b}}^{\mathrm{SI}, \mathrm{M}}$. As can be seen from FIG. 2(f) our potentials significantly underestimate the magnitude of this value for the $4 \mathrm{~d}$ and $5 \mathrm{~d}$ elements. Including this value in the fits did result in a more accurate reproduction but at significant cost to the reproduction of the other fit targets. Despite this the mixed site is consistently highly unfavoured. Thus the 
incorrect energy is unlikely to have serious consequences for any molecular dynamics simulations using these potentials, since the mixed dumbbell site is not a migration barrier. The interstitial is repelled by the solute by well above any realistic thermal energy and so the mixed interstitial site will occur with very low probability. Only for the $3 \mathrm{~d}$ solutes is the binding energy low enough to significantly influence solute dynamics. In this case our potentials perform well, even reproducing the positive binding of chromium and manganese in the mixed interstitial, despite being excluded from the fit.

To address the issue of the transferability of our potentials more generally it is worth first mentioning that the set of ab-initio configurations we have used in our fits act as examples of defect-free and undercoordinated and overcoordinated defect environments for the solute atoms. As such they act as computationally accessible prototypes for solute behaviour in similar environments near larger scale topological defects such as voids and dislocations. It is fair to say that our fitted potentials should therefore provide a similar degree of transferability.

In the absence of further ab-initio data to that discussed already we have used the following procedure in order to provide some measure of the transferability of our potentials. We have selected one representative element from each series, namely manganese, molybdenum and rhenium. For each element we remove each fit target in turn and refit the potential. The change in the omitted fit target from the full fit provides a measure of the likely uncertainty with which similar defects will be reproduced by the potential. We expect other elements in the same series to behave similarly. The results of this analysis are given in TABLE. 1.

It is clear that $E_{\mathrm{S}}$ and $E_{\mathrm{b}}^{\mathrm{SI}, \mathrm{C}}$ exhibit the largest changes after refitting. This is not too surprising given that the substitution energy is the only fit target directly associated with absolute energy values and given the problems already described with the instability of $E_{\mathrm{b}}^{\mathrm{SI}, \mathrm{C}}$. It is therefore necessary to include these values in the fits in order to reproduce them and, by implication, associated values well. The others, except $E_{\mathrm{b}}^{\mathrm{SI}, \mathrm{T}}$ for rhenium, change by less than the likely uncertainties in the fit target values themselves. This gives us confidence that similar quantities, not included in our fits, will be reproduced to similar accuracy and therefore in the overall transferability of our potentials. 


\section{Page 11 of 19

The question of transferability also arises with respect to the trends in the model values and rescaling parameters across a series i.e. is it possible to predict the parameters and therefore model values from those of its neighbouring elements. We answer this by plotting the model values produced by the rescale parameter functions (EQ. B1) in FIG. 4 in an identical manner to those for the full fits in FIG. 2.

A direct comparison of these two figures shows that the model values and trends across the series are preserved well by the change to the rescale parameter functions for most solutes, with some notable exceptions. Those with $n_{d}=2,3$ still perform poorly and reproduce the target data less well than the full fit potentials. Manganese performs particularly poorly, which is unsurprising given how poorly the full fit parameters are reproduced by the rescale parameter functions. The only other noteworthy change is that chromium is, perhaps, too strongly bound to a self-interstitial defect in the mixed and compressive sites. Once again it is the magnetic elements which give problems

Overall this shows that we can relate the rescale parameters back to the elementary electronic properties of the solute and still preserve the model values and trends across the transition metal series. This gives us confidence that we are capturing some of the relevant physics of these solutes through our simple rescaling strategy. We can also, in some sense, predict the properties of a particular solute from that of its neighbours through the rescale parameter functions and this demonstrates transferability between different solutes in a series.

\section{Solute-solute interactions in iron}

We have fitted the rescale parameters, $\left\{p_{i}^{(X, X)}\right\}$, to reproduce our ab-initio values [16] for the solute-solute binding energies from 1 nn to 5 nn separation, $E_{\mathrm{b}}^{X-X, i \mathrm{nn}}$, and to the separations between solutes at 1 nn separation, $r_{1 \mathrm{nn}}^{X-X}$, and $2 \mathrm{nn}$ separation, $r_{2 \mathrm{nn}}^{X-X}$ (see FIG. 5 for configurations). Our potential model values have been calculated in the same way as for the fits to the solute-iron rescale parameters and the same weight factors were used. The resulting values for the rescale parameters are shown in FIG. 6 and given in TABLE. B2, response function values are given in TABLE. B3 and our model values are compared with the ab-initio fit targets in FIG. 7.

The fit targets in FIG. 7 once again show a trend across the group and are well reproduced by our potentials. The picture emerging from the fit parameters themselves in FIG. 6 is less clear. For elements above half-filling there are clear trends with principal quantum number and $n_{d}$. However, for $5 \mathrm{~d}$ elements with $n_{d}<5$ there is considerable scatter for $p_{2}^{(X, X)}$ and $p_{3}^{(X, X)}$. It appears that a longer-ranged $\phi$ can compensate for a stronger repulsion. Since this anomaly is not present in the fit targets in FIG. 7, it must be an artifact of the fitting process itself and may well be due to the presence of multiple minima in the response function.

We have attempted to find a relationship between the solute-solute and solute-iron rescale parameters. However, even a direct plot of $p_{i}^{(X, X)}$ against $p_{i}^{(X)}$ showed no clear patterns. A functional dependence between the rescale parameters was a pre- 


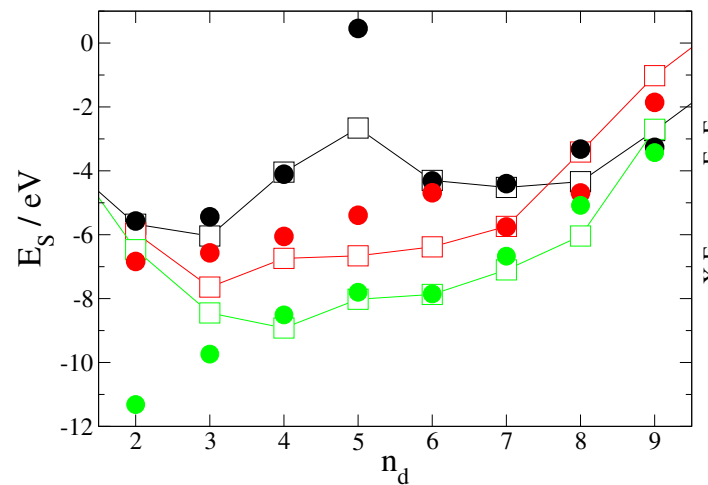

(a)

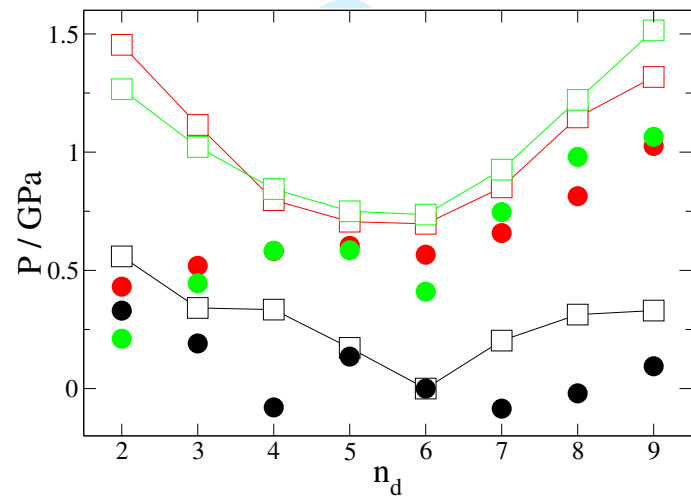

(c)

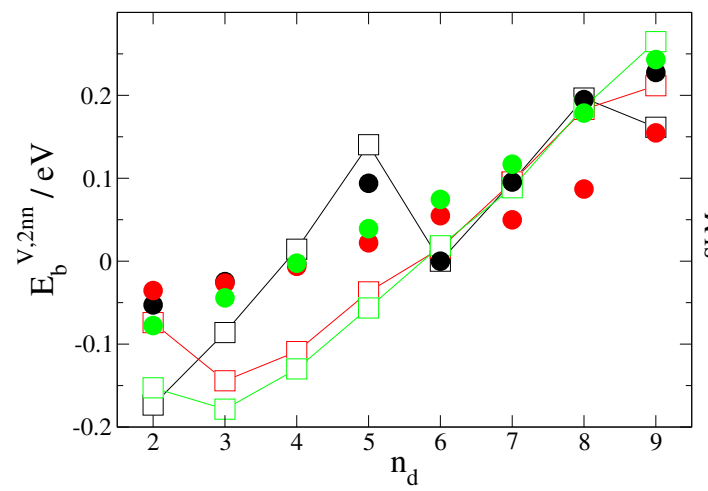

(e)

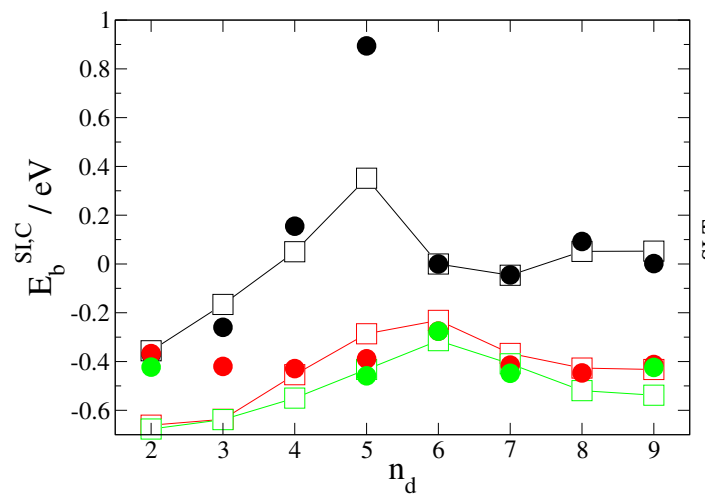

(g)

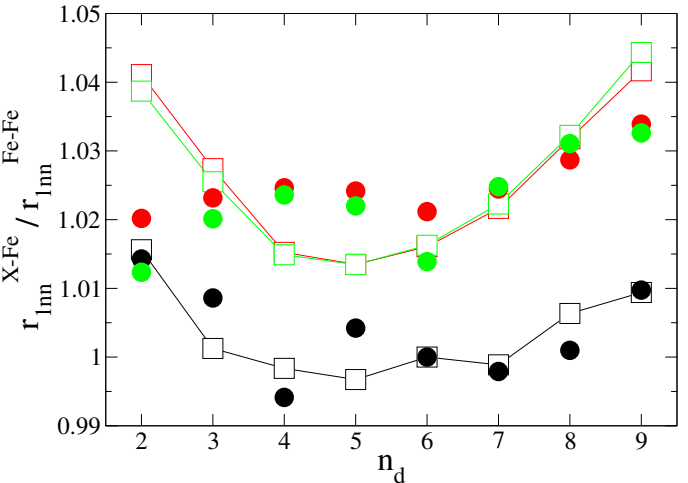

(b)

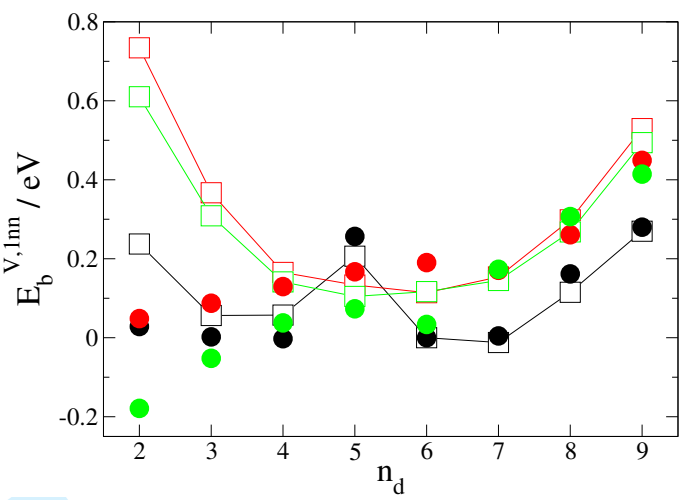

(d)

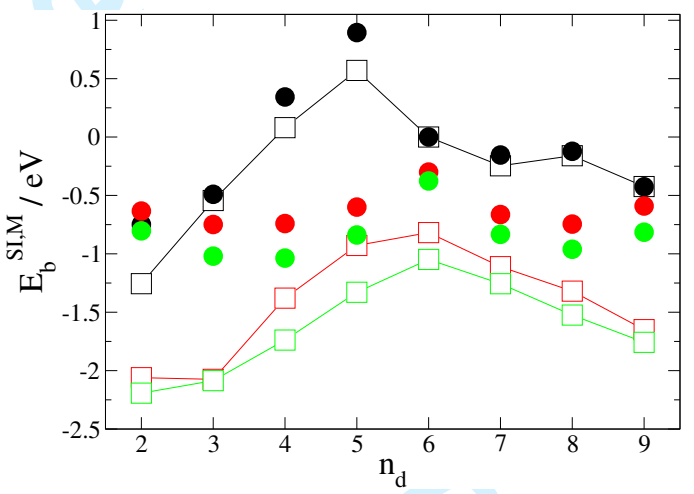

(f)

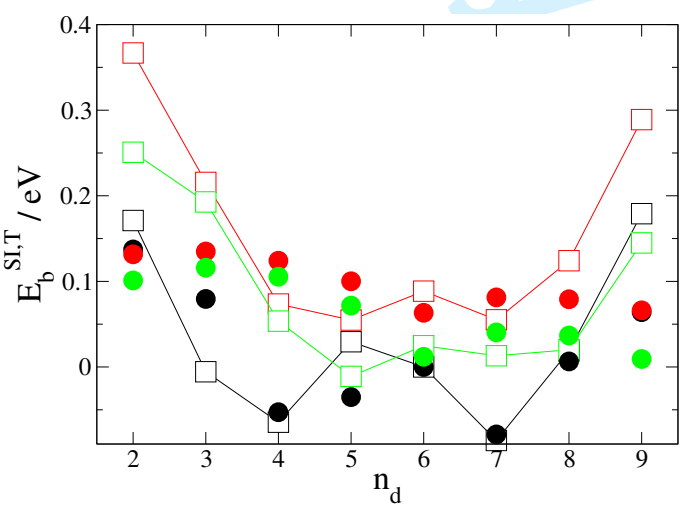

(h)

Figure 4. Fit targets and model values as in figure 2 but with model values generated from potentials

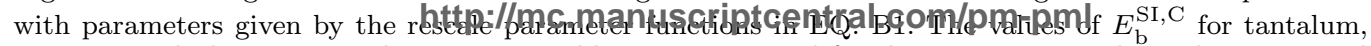
tungsten and platinum are absent as no stable minima existed for their parameter values: the interstitial simply relaxes away from the impurity. Colour versions are available online. 
Figure 5. Solute positions (black) in bcc iron (white) for configurations with two interacting solute particles. The numbered solute particles show the first to fifth nearest neighbour positions relative to the unnumbered solute.

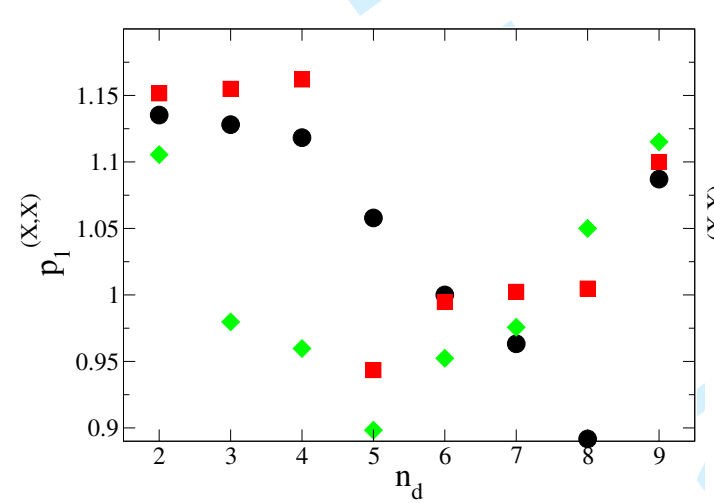

(a)

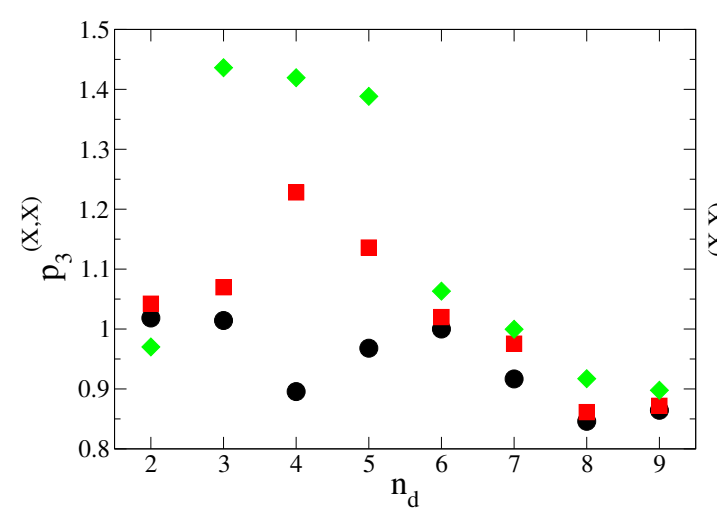

(c)

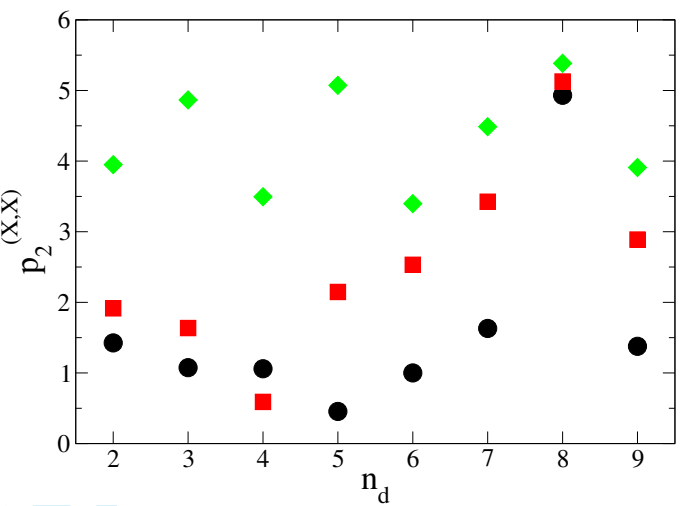

(b)

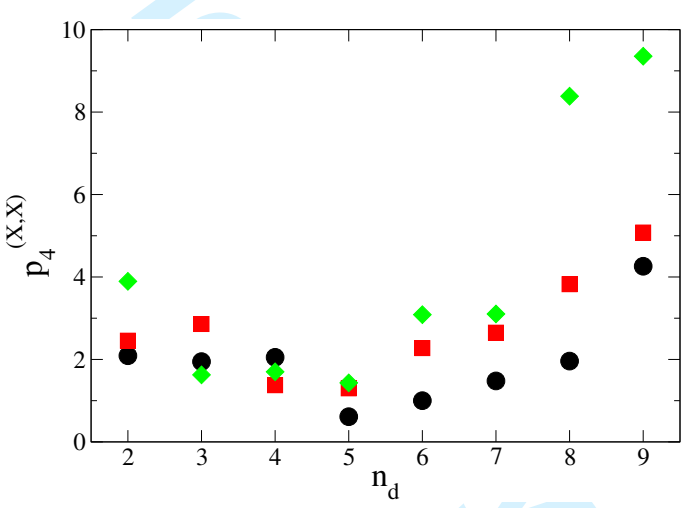

(d)

Figure 6. Rescale parameters as a function of the number of d-electrons for the 3d solutes (black circles), $4 \mathrm{~d}$ solutes (red squares) and $5 \mathrm{~d}$ solutes (green diamonds). The graphs are for (a) $p_{1}^{(X, X)},(\mathrm{b}) p_{2}^{(X, X)},(\mathrm{c})$ $p_{3}^{(X, X)}$ and $(\mathrm{d}) p_{4}^{(X, X)}$. Colour versions are available online. 


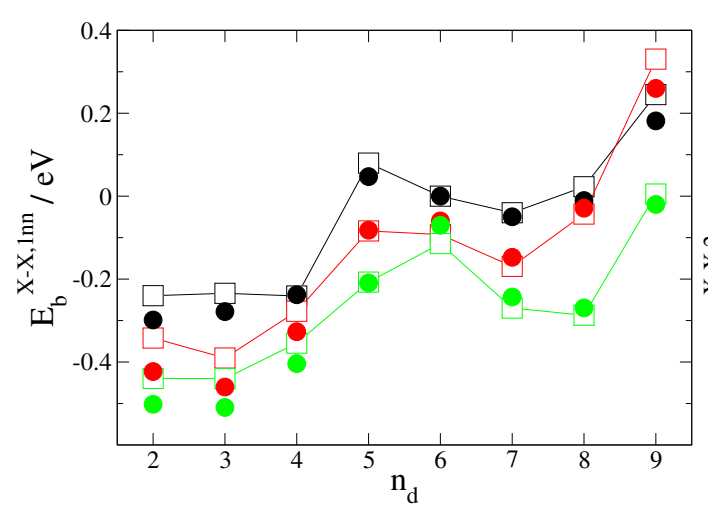

(a)

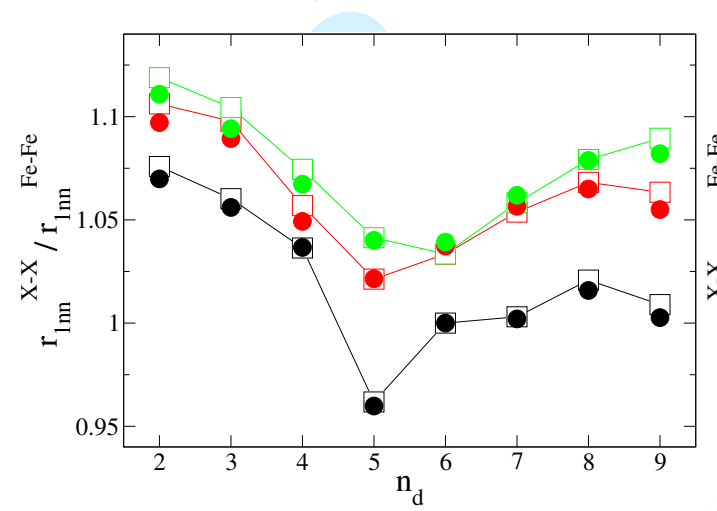

(c)

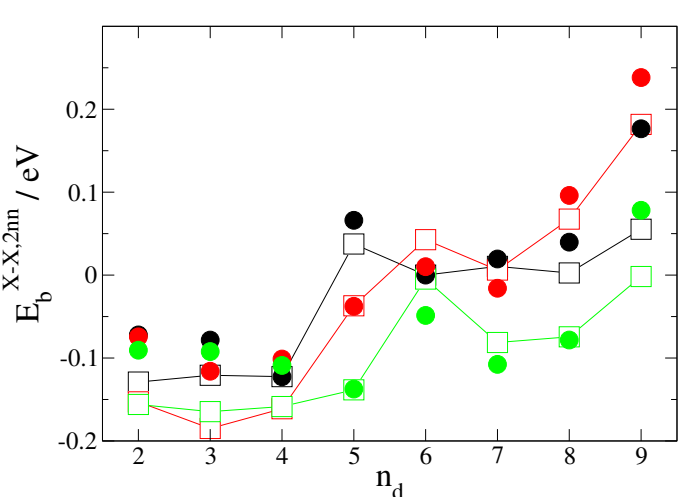

(b)

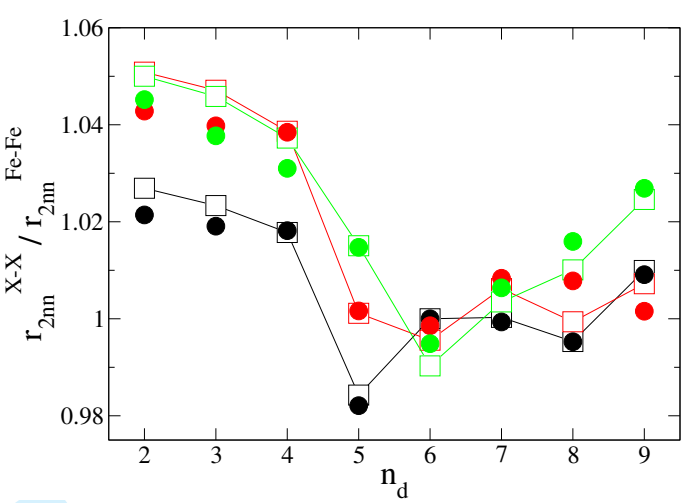

(d)

Figure 7. Solute-solute fit targets (squares and lines), derived from our ab-initio data, and corresponding values from our semi-empirical model (circles) versus number of d-electrons for $3 \mathrm{~d}$ solutes (black), 4d solutes (red) and 5d solutes (green). In particular we plot (a) the solute-solute binding energy at 1nn separation, (b) the solute-solute binding energy at $2 \mathrm{nn}$ separation, (c) the relative solute-solute $1 \mathrm{nn}$ separation and (d) the relative solute-solute $2 \mathrm{nn}$ separation. Colour versions are available online.

requisite for the construction of a multi-component potential based on rescaling factors $p_{i}^{(X, Y)}$ and so this has not been possible in the current work. We have, however, produced binary potentials for transition metal solutes in $\alpha$-iron from a simple rescaling technique, many of which reproduce the ab-initio data to within the likely precision of such calculations.

\section{Conclusions}

In conclusion, we have advanced the hypothesis that the interactions between transition metal atoms in iron can be described by simply scaling the parameters of a Finnis-Sinclair model, and further that these scaling parameters are simply functions of the number of $d$-electrons and the principal quantum number. Quantum mechanical calculations of interactions between solutes and point defects show clear trends across the group. We have presented best-fit rescaled potentials for all transition metal elements in an iron matrix. Furthermore we have been able to model the rescale parameters for iron-solute interactions, and subsequently the 


\section{Acknowledgements}

We gratefully acknowledge support from the EU FP7 GETMAT programme.

\section{References}

[1] M.W. Finnis and J.E. Sinclair, Phil. Mag. A 50, 45-55 (1984).

[2] Ducastelle and Cyrot-Lackmann Adv.Phys 16, 393 (1967); J.Phys.Chem.Solids, 31,1295 (1970)

[3] G. J. Ackland, M. W. Finnis, and V. Vitek, J. Phys. F 18, L153 (1988).

[4] G. J. Ackland, G. I. Tichy, V. Vitek, and M. W. Finnis, Phil. Mag. A, 56, 735 (1987).

[5] P. Olsson, J. Wallenius, C. Domain, K. Nordlund, L. Malerba, Phys. Rev. B 74, 229906 (2006).

[6] Bonny et al., submitted to Phil. Mag. Finnis-Sinclair special issue.

[7] M.S. Daw and M.I. Baskes, Phys. Rev. B 29, 6443-6453 (1984).

[8] R.A. Johnson, Phys. Rev. B 39, 12554-12559 (1989).

[9] G.J. Ackland and S.K. Reed, Phys. Rev. B 67, 174108 (2003).

[10] V.I. Anisimov, V.P. Antropov, A.I. Liechtenstein, V.A. Gubanov and A.V. Postnikov, Phys. Rev. B 37, 5598 (1988).

[11] B. Drittler, N. Stefanou, S. Blügel, R. Zeller and P.H. Dederichs, Phys. Rev. B 40, 8203(1989).

[12] G. Kresse and J. Hafner, Phys. Rev. B 47, 558 (1993); G. Kresse and J. Furthmuller, Phys. Rev. B 54, 11169 (1996).

[13] P.E. Blöchl, Phys. Rev. B 50, 17953 (1994); G. Kresse and D. Joubert, Phys. Rev. B 59, 1758 (1999).

[14] J.P. Perdew et al., Phys. Rev. B 46, 6671 (1992).

[15] S.H. Vosko, L. Wilk and M. Nusair, J. Can. Phys. 58, 1200 (1980).

[16] P.Olsson, E.Vincent and C.Domain, in preparation

[17] J.D. Cox, D.D. Wagman and V.A. Medvedev, Codata Key Values for Thermodynamics, Hemisphere Pub. Corp., New York, (1989).

[18] G.J. Ackland, D.J. Bacon, A.F. Calder and T. Harry, Phil. Mag. A 75, 713-732 (1997).

[19] M.I. Mendelev et al., Phil. Mag. 83, 3977-3994 (2003).

[20] G.J. Ackland, M.I. Mendelev, D.J. Srolovitz, S. Han and A.V. Barashev, J. Phys.:Condens. Matter 16, S2629-S2642 (2004).

[21] M. Müller, P. Erhart and K. Albe, J.Phys.:Condens.Mater 19, 326220 (2007).

$[22]$ J.P. Biersack and J.F. Ziegler, Nucl. Instrum. Methods 194, 93-100 (1982).

[23] V. Torczon, SIAM J. Optim. 7, 1-25 (1997).

\section{Appendix A. Pure iron potential}

The parameters for the pure iron potential of Ackland et al. [18] are given in TABLE. A1. It is worth pointing out that the function, $\phi(r)$, with these parameters actually has a maximum value at $r_{\max }=1.82021002 \mathrm{~A}^{\circ}$ of $\phi\left(r_{\max }\right)=3.222480407 \mathrm{eV}^{2}$ 
Table A1. Parameters for the pure iron potential [18].

\begin{tabular}{lll}
\hline & $a_{k}^{\mathrm{Fe}, \mathrm{Fe}} / \mathrm{eVA}^{\circ}-3$ & $r_{k}^{(\mathrm{Fe}, \mathrm{Fe})} / \AA$ \\
\hline 1 & -1.5522033 & 3.38247 \\
2 & 2.649964948 & 3.296475 \\
3 & -0.558542776 & 3.09582 \\
4 & -0.115540092 & 2.837835 \\
5 & 0.372003235 & 2.665845 \\
6 & 4.245649731 & 2.482460633 \\
\hline & $A_{k}^{\mathrm{Fe}, \mathrm{Fe}} / \mathrm{eV}^{2} \AA^{-3}$ & $R_{k}^{(\mathrm{Fe}, \mathrm{Fe})} / \AA$ \\
\hline 1 & 3.093735585 & 3.72645 \\
2 & -4.285763266 & 3.4398 \\
\hline
\end{tabular}

and becomes negative for low separations. To avoid any possible problems this may cause the author recommends setting $\phi$ to its maximum value below $r_{\text {max }}$. Such radii are only sampled under extreme conditions such as high pressures or in radiation damage simulations and this change will not modify any equilibrium or near equilibrium properties of the iron model.

Appendix B. Rescale parameters and fit results

The fitted values for the rescale parameters for iron-solute, $p_{i}^{(X)}$, and solute-solute, $p_{i}^{(X, X)}$, interactions are given in TABLES. B1 and B2. The response function values for the respective fits are given in TABLE. B3. The equations for the rescale parameter functions are shown in EQ. B1. 


\section{Page 17 of 19 \\ Philosophical Magazine \& Philosophical Magazine Letters}

REFERENCES

Table B1. Rescale parameters for iron-solute interactions

\begin{tabular}{ccccc}
\hline Solute, $X$ & $p_{1}^{(X)}$ & $p_{2}^{(X)}$ & $p_{3}^{(X)}$ & $p_{4}^{(X)}$ \\
\hline $\mathrm{Ti}$ & 1.1079352022752937 & 0.69914888963867840 & 1.0084290227415416 & 1.5946911193111377 \\
$\mathrm{~V}$ & 1.0704687944688829 & 0.68703519774868570 & 1.0084191748980367 & 1.4652839386546592 \\
$\mathrm{Cr}$ & 1.0504446925177677 & 0.68660606895558630 & 0.9784540205196225 & 1.1287695242404665 \\
$\mathrm{Mn}$ & 0.8727971294583414 & 2.48881963381730960 & 1.1228404871022541 & 0.3065915687995297 \\
$\mathrm{Co}$ & 1.0467024050842750 & 0.94717978096230060 & 0.9320535734737820 & 1.7396896806120596 \\
$\mathrm{Ni}$ & 1.1194809316877880 & 0.92438826748289380 & 0.8883635622171985 & 3.4100636005122293 \\
$\mathrm{Cu}$ & 1.1208802828524023 & 0.98505124753645820 & 0.8765649836565008 & 3.2065254293566600 \\
\hline $\mathrm{Zr}$ & 1.2841655075134310 & 0.40739377742609160 & 0.9860071367651349 & 2.7482154017401133 \\
$\mathrm{Nb}$ & 1.2841655075134310 & 0.40336016805208613 & 0.9909619464976229 & 3.0647879434909940 \\
$\mathrm{Mo}$ & 1.2841655075134310 & 0.40306261907883430 & 0.9909619275965241 & 3.0437240549628903 \\
$\mathrm{Tc}$ & 1.2714509975380506 & 0.37927270590314116 & 0.9860317313257698 & 2.8186046924493455 \\
$\mathrm{Ru}$ & 1.2932752539714167 & 0.33285431259332965 & 0.9918471983137220 & 2.7002031043018238 \\
$\mathrm{Rh}$ & 1.3583482886258411 & 0.36634462066369505 & 0.9706236772142814 & 3.8933887256877090 \\
$\mathrm{Pd}$ & 1.3551721040070680 & 0.39024726568702440 & 0.9674492344138608 & 3.3285717131180084 \\
$\mathrm{Ag}$ & 1.4023136316534160 & 0.35592505640677500 & 0.9705185530509607 & 2.9551289346288447 \\
\hline $\mathrm{Hf}$ & 1.2841655075134310 & 0.40746747208599430 & 0.9860071367651349 & 2.7521572914348287 \\
$\mathrm{Ta}$ & 1.3381889417068988 & 0.42350948842405384 & 0.9734862986585047 & 4.8610714605878340 \\
$\mathrm{~W}$ & 1.3381889417068988 & 0.42350948842405384 & 0.9734862986585047 & 4.8610567185373155 \\
$\mathrm{Re}$ & 1.3381889417068988 & 0.41247111384741486 & 0.9686430832422932 & 4.6812582679644960 \\
$\mathrm{Os}$ & 1.3963331257722620 & 0.33347841442944126 & 0.9760396335905974 & 4.7084791631263130 \\
$\mathrm{Ir}$ & 1.3825438675169937 & 0.40034598076904200 & 0.9633439996351745 & 5.2329577841196330 \\
$\mathrm{Pt}$ & 1.4045418753797260 & 0.41926814851469420 & 0.9633439996351745 & 5.4900896361876255 \\
$\mathrm{Au}$ & 1.4447702630891220 & 0.40420070742435266 & 0.9585272796369986 & 5.1583042920958950 \\
\hline & & & &
\end{tabular}

$p_{1}^{(3 \mathrm{~d})}\left(n_{d}\right)=0.93252041+\left\{\begin{aligned}-0.12134445\left(n_{d}-5\right)-0.02163213\left(n_{d}-5\right)^{2}, & n_{d}<5 \\ 0.07352920\left(n_{d}-5\right)-0.00604960\left(n_{d}-5\right)^{2}, & n_{d} \geq 5\end{aligned}\right.$

$p_{2}^{(3 \mathrm{~d})}\left(n_{d}\right)= \begin{cases}0.69093005 & \\ 2.52957942-0.42093634 n_{d}+0.02766774 n_{d}^{2}, & n_{d}<5 \\ n_{d} \geq 5\end{cases}$

$p_{3}^{(3 \mathrm{~d})}\left(n_{d}\right)= \begin{cases}1.04339658-0.01498750 n_{d} & , n_{d}<5 \\ 2.25879719-0.32320567 n_{d}+0.01890102 n_{d}^{2}, & n_{d} \geq 5\end{cases}$

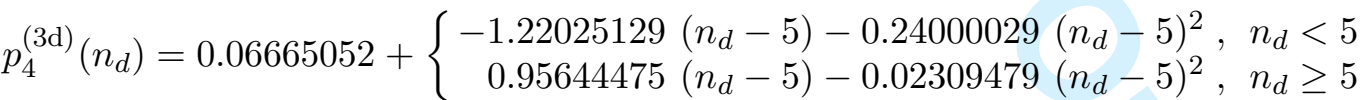

$p_{1}^{(4 \mathrm{~d})}\left(n_{d}\right)=1.22323408+0.01698146 n_{d}$

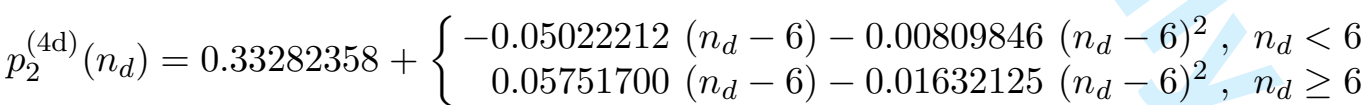

$p_{3}^{(4 \mathrm{~d})}\left(n_{d}\right)=1.00021096-0.00334742 n_{d}$

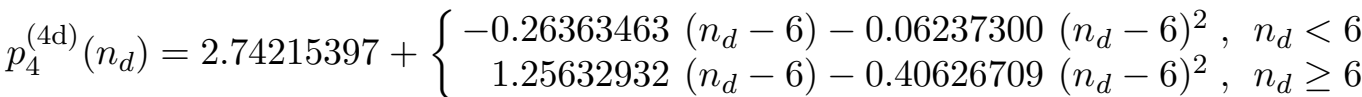

$p_{1}^{(5 \mathrm{~d})}\left(n_{d}\right)=1.25801235+0.01960961 n_{d}$

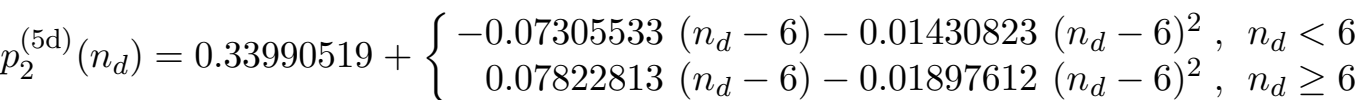

$p_{3}^{(5 \mathrm{~d})}\left(n_{d}\right)=0.98778299-0.00316787 n_{d}$

$p_{4}^{(5 \mathrm{~d})}\left(n_{d}\right)=4.66010696+\left\{\begin{aligned}-0.12504119\left(n_{d}-6\right)-0.02240603\left(n_{d}-6\right)^{2} & , n_{d}<6 \\ 0.83855171\left(n_{d}-6\right)-0.22256433\left(n_{d}-6\right)^{2} & , n_{d} \geq 6\end{aligned}\right.$ 
Table B2. Rescale parameters for solute-solute interactions

\begin{tabular}{cllll}
\hline Solute, $X$ & \multicolumn{1}{c}{$p_{1}^{(X, X)}$} & \multicolumn{1}{c}{$p_{2}^{(X, X)}$} & \multicolumn{1}{c}{$p_{3}^{(X, X)}$} & \multicolumn{1}{c}{$p_{4}^{(X, X)}$} \\
\hline $\mathrm{Ti}$ & 1.135234375 & 1.425375 & 1.0184375 & 2.090625 \\
$\mathrm{~V}$ & 1.128015625 & 1.07515625 & 1.01421875 & 1.9471875 \\
$\mathrm{Cr}$ & 1.1182627956431268 & 1.0591971435622631 & 0.8956061196601681 & 2.0520677210215306 \\
$\mathrm{Mn}$ & 1.0578763126373347 & 0.45488252197265816 & 0.9680164178466799 & 0.6121371734619128 \\
$\mathrm{Co}$ & 0.9632660156249979 & 1.63034375 & 0.916725 & 1.47909375 \\
$\mathrm{Ni}$ & 0.8917071437428479 & 4.931618183795954 & 0.846 & 1.9593257026672335 \\
$\mathrm{Cu}$ & 1.087078125 & 1.3775 & 0.864421875 & 4.26 \\
\hline $\mathrm{Zr}$ & 1.1515625 & 1.915 & 1.04225 & 2.455375 \\
$\mathrm{Nb}$ & 1.155 & 1.63625 & 1.06975 & 2.859375 \\
$\mathrm{Mo}$ & 1.16200390625 & 0.590203125 & 1.228046875 & 1.374328125 \\
$\mathrm{Tc}$ & 0.94346875 & 2.1465625 & 1.135921875 & 1.299375 \\
$\mathrm{Ru}$ & 0.9946875 & 2.5315625 & 1.0199765625 & 2.275625 \\
$\mathrm{Rh}$ & 1.00234375 & 3.425 & 0.9755625 & 2.648625 \\
$\mathrm{Pd}$ & 1.00478125 & 5.126925 & 0.8613123046875 & 3.8247 \\
$\mathrm{Ag}$ & 1.1 & 2.889 & 0.871875 & 5.07775 \\
\hline $\mathrm{Hf}$ & 1.1055 & 3.95 & 0.9700625 & 3.894 \\
$\mathrm{Ta}$ & 0.97971796875 & 4.86585 & 1.436123959960937 & 1.625 \\
$\mathrm{~W}$ & 0.9597262573242189 & 3.4952026367187496 & 1.4194162597656252 & 1.6996997070312496 \\
$\mathrm{Re}$ & 0.8983463569335935 & 5.072334990624995 & 1.388288172737312 & 1.431971765625 \\
$\mathrm{Os}$ & 0.952375 & 3.3975 & 1.0632097695312475 & 3.085089 \\
$\mathrm{Ir}$ & 0.9756723193359381 & 4.48695 & 0.9996396093750014 & 3.10280625 \\
$\mathrm{Pt}$ & 1.05 & 5.385 & 0.917125 & 8.385 \\
$\mathrm{Au}$ & 1.115125 & 3.90925 & 0.89775 & 9.355 \\
\hline & & & & \\
\hline
\end{tabular}


Table B3. Response function values, $\chi^{2}$, for the solute-iron rescale parameters given in TABLE. B1 and the solute-solute rescale parameters given in TABLE. B2.

\begin{tabular}{crr}
\hline Solute, $X$ & $\chi^{2}(X-\mathrm{Fe})$ & $\chi^{2}(X-X)$ \\
\hline $\mathrm{Ti}$ & 568.967 & 96.770 \\
$\mathrm{~V}$ & 221.205 & 58.907 \\
$\mathrm{Cr}$ & 99.636 & 33.934 \\
$\mathrm{Mn}$ & 160.962 & 23.567 \\
$\mathrm{Co}$ & 43.902 & 2.755 \\
$\mathrm{Ni}$ & 65.728 & 32.237 \\
$\mathrm{Cu}$ & 204.809 & 204.468 \\
\hline $\mathrm{Zr}$ & 4796.904 & 284.391 \\
$\mathrm{Nb}$ & 1689.753 & 208.931 \\
$\mathrm{Mo}$ & 216.139 & 88.197 \\
$\mathrm{Tc}$ & 137.204 & 9.579 \\
$\mathrm{Ru}$ & 69.618 & 36.145 \\
$\mathrm{Rh}$ & 26.411 & 104.771 \\
$\mathrm{Pd}$ & 103.652 & 61.308 \\
$\mathrm{Ag}$ & 477.687 & 142.646 \\
\hline $\mathrm{Hf}$ & 3491.843 & 182.800 \\
$\mathrm{Ta}$ & 1502.974 & 152.638 \\
$\mathrm{~W}$ & 408.336 & 76.466 \\
$\mathrm{Re}$ & 198.776 & 1.503 \\
$\mathrm{Os}$ & 85.532 & 261.420 \\
$\mathrm{Ir}$ & 35.811 & 284.450 \\
$\mathrm{Pt}$ & 69.882 & 302.596 \\
$\mathrm{Au}$ & 386.018 & 186.895 \\
\hline & &
\end{tabular}

